\title{
Cosmogenic Radionuclides as an Extension of the Neutron Monitor Era into the Past: Potential and Limitations
}

\author{
J. Beer • K.G. McCracken • J. Abreu • U. Heikkilä • \\ F. Steinhilber
}

Received: 13 September 2011 / Accepted: 30 September 2011 / Published online: 1 December 2011

(C) Springer Science+Business Media B.V. 2011

\begin{abstract}
The cosmogenic radionuclides, ${ }^{10} \mathrm{Be},{ }^{14} \mathrm{C}$ and others, provide a record of the paleo-cosmic radiation that extends $>10,000$ years into the past. They are the only quantitative means at our disposal to study the heliosphere prior to the commencement of routine sunspot observations in the 17th century. The cosmogenic radionuclides are primarily produced by secondary neutrons generated by the galactic cosmic radiation, and can be regarded, in a sense, as providing an extrapolation of the neutron monitor era into the past. However, their characteristics are quite different from the man-made neutron monitor in several important respects: (1) they are sensitive to somewhat lower cosmic ray energies; (2) their temporal resolution is $\sim 1$ to 2 years, being determined by the rapidity with which they are sequestered in ice, biological, or other archives; (3) the statistical precision for annual data is very poor $(\sim 19 \%)$; however it is quite adequate $(\sim 5 \%$ for 22 -year averages) to study the large variations $( \pm 40 \%)$ that have occurred in the paleo-cosmic ray record in the past between grand solar minima and maxima. The data contains "noise" caused by local meteorological effects, and longer-term climate effects, and the use of principal component analysis to separate these "system" effects from production effects is outlined. The concentrations of ${ }^{10} \mathrm{Be}$ decreased by a factor of two at the commencement of Holocene, the present-day "interglacial", due to a $100 \%$ increase in the ice accumulation rates in polar regions. The use of the ${ }^{10} \mathrm{Be}$ flux to study heliospheric properties during the last glacial is discussed briefly.
\end{abstract}

Keywords Cosmogenic radionuclides $\cdot$ Cosmic rays $\cdot$ Neutron monitors $\cdot$ Solar activity

J. Beer $(\varangle) \cdot$ J. Abreu $\cdot$ F. Steinhilber

Eawag, Dübendorf, Switzerland

e-mail: juerg.beer@eawag.ch

K.G. McCracken

Institute of Physical Science and Technology, University of Maryland, College Park, Maryland, USA

U. Heikkilä

Australian Nuclear Science and Technology Organisation (ANSTO), Lucas Heights, NSW, Australia 


\section{Introduction}

The cosmic radiation provides a window to space that reveals unique information about the generation of the galactic cosmic radiation (GCR) by supernovae, their propagation through the galaxy, and their modulation by the heliomagnetic and geomagnetic fields. Following the discovery of the cosmic radiation by Hess in 1912, its intensity was measured in terms of the ionisation it causes when it interacts with a gas. Ionization chambers were later replaced by dedicated devices specifically designed to only record neutrons, the so-called neutron monitors (NM). The first NM was established at Climax, Colorado in 1951 and by 1957 there was a continuous worldwide monitoring network of NM, which now numbers about 60 instruments.

These man-made NM have been complemented by the development of the means to decipher the data from a natural NM which has been recording the cosmic ray induced neutrons far into the distant past. The cosmic rays interact with atoms in the atmosphere producing a variety of radioactive isotopes, such as ${ }^{14} \mathrm{C}$ and ${ }^{10} \mathrm{Be}$, called the cosmogenic radionuclides. The development of accelerator mass spectrometry in the 1980s increased the detection limit by orders of magnitude such that the cosmogenic radionuclides can now be measured in very small quantities. They are complementary to the man-made NM in the sense that they cover multi-millennial instead of multi-decadal time periods, however with a much lower temporal resolution (years compared to minutes) and with much less statistical precision. Throughout this paper we will discuss the use of the cosmogenic radionuclides as measurements of the "paleo-cosmic radiation".

The main goal of this paper is to discuss the physical basis of the natural NM and to give an overview of its potential and limitations.

\section{Counting Techniques}

A man-made NM contains of a number of proportional counters filled with a few litres of counting gas. It is designed to be sensitive to high-energy neutrons (Simpson 2000). An incoming high-energy neutron is identified unambiguously in real time by the characteristics of the electrical charge pulse it produces in the counters.

In the case of the natural NM the counter is the whole atmosphere, and the counting gas is air. Besides the much larger size of the counting volume, the fundamental difference is that a natural NM does not count the very short-lived ionization process in real time; it produces and sequesters the reaction products, the cosmogenic radionuclides, that are generated by the nuclear interactions. Since the main components of air in weight percent are $\mathrm{N}$ (75.51\%), $\mathrm{O}(23.15 \%)$, and $\mathrm{Ar}(1.28 \%)$ the majority of the useful cosmogenic radionuclides have masses that are smaller than 40 . Restricting our attention to those with half-lives longer than $100 \mathrm{y}$ the list is relatively short (Table 1).

The cosmogenic radionuclides are produced by the proton and neutron secondaries of the primary cosmic rays. The third column of Table 1 shows the calculated mean global production rates for the present geomagnetic dipole moment and the solar modulation potential $\phi=550 \mathrm{MV}$ (Masarik and Beer 2009). The percentage of each radionuclide produced by neutrons is given in brackets. To estimate the dependence of the production rate on latitude and altitude the atmosphere was divided into $10^{\circ}$ latitudinal bands and altitudinal layers of $30 \mathrm{~g} \mathrm{~cm}^{-2}$. Using the GEANT code, the production rates were simulated for the 306 atmospheric bins, for different dipole moments and solar modulation potentials. 
Table 1 Mean global production rates of the main cosmogenic radionuclides with half-lives longer than $100 \mathrm{y}$ that are produced in the atmosphere for $\phi=550 \mathrm{MV}$ and the present geomagnetic field. The relative contribution by neutrons is given in brackets

\begin{tabular}{lll}
\hline Nuclide & Half-life (kyr) & Prod. Rate $\left(\mathrm{cm}^{-2} \mathrm{~s}^{-1}\right)$ \\
\hline${ }^{10} \mathrm{Be}$ & 1,370 & $0.021(81 \%)$ \\
${ }^{14} \mathrm{C}$ & 5.730 & $2.02(100 \%)$ \\
${ }^{26} \mathrm{Al}$ & 710 & $4.70 \times 10^{-5}(49 \%)$ \\
${ }^{32} \mathrm{Si}$ & 0.14 & \\
${ }^{36} \mathrm{Cl}$ & 301 & $1.12 \times 10^{-3}(68 \%)$ \\
${ }^{39} \mathrm{Ar}$ & 0.269 & \\
\hline
\end{tabular}

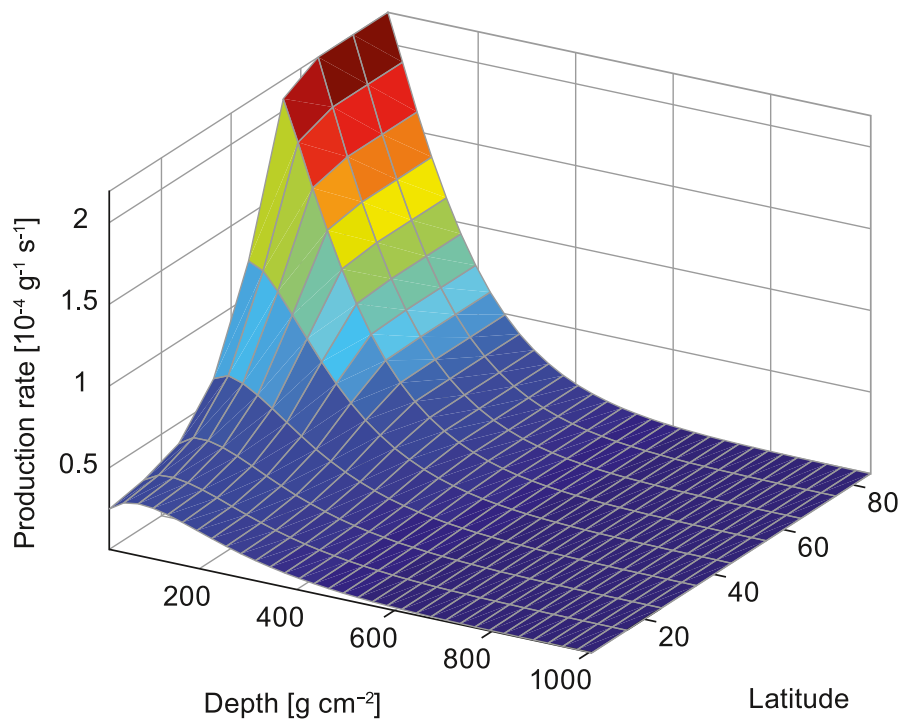

Fig. $1{ }^{10}$ Be production rate per gram of air as a function of geomagnetic latitude and atmospheric depth in $\mathrm{g} \mathrm{cm}^{-2}$ for the present geomagnetic dipole field and a solar modulation potential $\phi=550 \mathrm{MV}$

The dependence upon geomagnetic latitude and atmospheric depth is shown in Fig. 1. As a result of the atmospheric and geomagnetic shielding effects the production is largest at high altitudes and high latitudes. Figure 2 depicts the dependence of the mean global production rate of ${ }^{10} \mathrm{Be}$ on the dipole field in units relative to the present value and the solar modulation potential $\phi$. The dependence of the other radionuclides upon these factors is similar.

The full dynamic range of the ${ }^{10} \mathrm{Be}$ production rate between a quiet sun $(\phi=0 \mathrm{MV})$ and no dipole moment $(M=0)$ to a very active sun $(\phi=1,000 \mathrm{MV})$ and a doubling of the present dipole moment is almost an order of magnitude.

Figure 3 presents the response functions of a number of different cosmic ray detectors to primary cosmic ray protons. ${ }^{10} \mathrm{Be}$ is mainly sensitive to primary particles between 0.5 and 3 $\mathrm{GeV} /$ nucleon, depending in part on the level of solar modulation. The sensitivity of a typical man-made neutron monitor at sea level peaks at a higher energy of $\sim 4.5 \mathrm{GeV} /$ nucleon. As a result, the amplitude of the 11-year Schwabe cycle in the ${ }^{10} \mathrm{Be}$ data is a factor of $\sim 2.3$ greater than in the case of the man-made NM. 


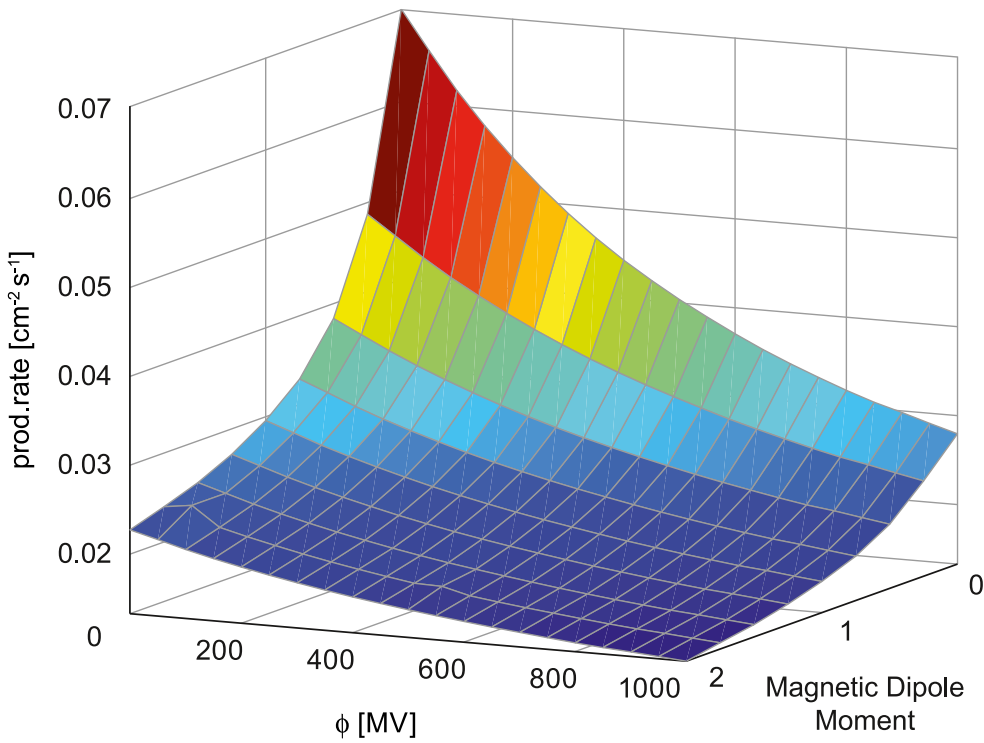

Fig. 2 Mean global ${ }^{10}$ Be production rate as a function of solar modulation and geomagnetic dipole moment (in units relative to today)

Fig. 3 Comparison of the response functions for the natural neutron monitor ${ }^{10} \mathrm{Be}$ with a man-made neutron monitor at sea level (McCracken 2004)

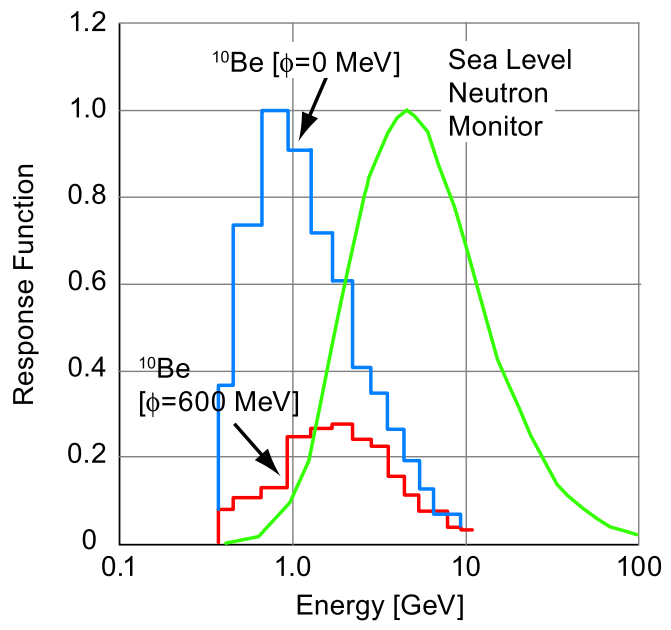

\section{Recording}

In the previous section we have seen that the production rate of cosmogenic radionuclides in the past has been determined by the GCR intensity at that time. We now address the question of how nature has recorded that production rate in archives which we can access and interpret today. For this purpose we consider the fate of the cosmogenic radionuclides after production somewhere in the atmosphere. First of all, their fate depends strongly on their geochemical properties. For example, ${ }^{10} \mathrm{Be}$ attaches quickly to aerosols and subsequently follows the relatively short-lived aerosol pathways in the atmosphere. In stark contrast, ${ }^{14} \mathrm{C}$ oxidizes to ${ }^{14} \mathrm{CO}_{2}$ and enters the carbon cycle. This means that ${ }^{14} \mathrm{C}$ exchanges between the main 
Fig. 4 Zonally averaged mean residence time of aerosols as a function of latitude and altitude in hPa (Shepherd 2007)

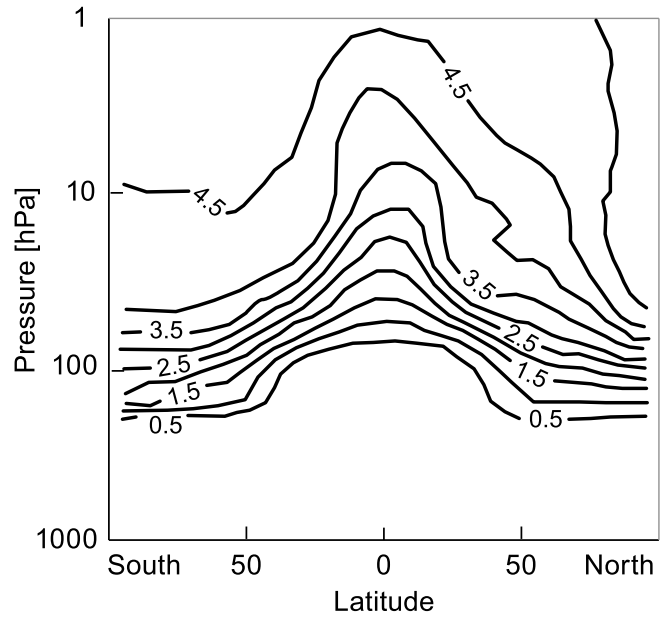

components of the carbon cycle, the atmosphere, the biosphere, and the ocean, and a ${ }^{14} \mathrm{C}$ atom may not enter an archive for many centuries. ${ }^{10} \mathrm{Be}$ and other aerosol bound nuclides such as ${ }^{7} \mathrm{Be},{ }^{26} \mathrm{Al},{ }^{32} \mathrm{Si}$, and ${ }^{36} \mathrm{Cl}$ are removed from the atmosphere within several years mainly by wet precipitation (rain and snow). For this to happen, the cosmogenic radionuclide bearing aerosols must reach the lower troposphere. As Fig. 1 shows, most of the cosmogenic radionuclide production $(\sim 65 \%)$ takes place in the stratosphere, and the time needed to reach the Earth's surface is strongly altitude dependent. Figure 4 shows the mean zonally averaged stratospheric residence time for aerosols is in the range 1-4 years.

As a consequence it can take several years for a nuclide to pass through the tropopause and reach the lower troposphere. It is important to note that the height of the tropopause is latitude and time dependent and that the exchange occurs in the mid-latitude mainly during the local spring to summer. A nuclide produced in the troposphere is removed by snow and rain in a period ranging between days and a few weeks.

As we will discuss in the next section these transport processes are much more complex than outlined above (Holton et al. 1995), and they introduce two major limitations of the paleo-cosmic ray record (i.e., limited temporal and spatial resolution).

In order to record the history of cosmic ray intensity the cosmogenic radionuclides produced in the atmosphere must be stored in a natural archive in a stratigraphically undisturbed way. Since ${ }^{10} \mathrm{Be}$ and other aerosol bound cosmogenic radionuclides are mainly removed from the atmosphere by rain and snow, ice is the archive of first choice. Snowflakes carrying cosmogenic radionuclides are deposited in the polar regions and without any further disturbances they slowly turn into ice. In this way cosmogenic radionuclides are stored for up to a million year in the ice sheets in Antarctica. One reason for this age limit is that the weight of the overlying layers causes ice to flow slowly towards the coast, and as a consequence the thickness of an annual ice layer decreases with increasing depth, leading to a strongly nonlinear depth-age relationship. Lake and deep sea sediments provide another archive which has the potential to go back many millions of years, however at the expense of a high temporal resolution. The sedimentation rate is in the order of $1 \mathrm{~mm}$ per year in lakes, while in the sea it is about a thousand times smaller. The difficulty in using sediments is that the transfer from the atmosphere into the sediment introduces an additional filter which affects the production signal. Erosion from the catchment can bring in additional cosmogenic radionuclides and the concentration of particles in the water, and the geochemical conditions, influences the local removal rate of cosmogenic radionuclides. 
${ }^{14} \mathrm{C}$ is incorporated into plants by photosynthesis. Tree rings therefore provide an ideal archive for ${ }^{14} \mathrm{C}$, and by combining the data from trees with overlapping ages (dendrochronology), this record can be extended from the present to 50,000 years in the past (this limit is set by the ${ }^{14} \mathrm{C}$ half-life of 5,370 y). At present dendrochronology is limited to about 12,400 years (Reimer et al. 2009), but it is expected to ultimately reach 50,000 years in coming years. The storage of ${ }^{14} \mathrm{C}$ in the oceans and biosphere can be represented by a mathematical filter (Siegenthaler and Beer 1988), and this shows that the observed variations in ${ }^{14} \mathrm{C}$ in tree rings suffer frequency dependent attenuation. Thus while the 11 year variation in cosmic ray intensity results in a $\sim 40 \%$ variation in the production of ${ }^{14} \mathrm{C}$, the observed variation in tree rings is attenuated by a factor of $\sim 100$. Mathematical techniques are now used to invert the observed ${ }^{14} \mathrm{C}$ data to yield the original variations in production rate (see Chap. 13, Beer et al. 2011).

Some ice cores extend $>100,000$ yrs into the past. They are cut into annual or longer samples, and then the concentration of the radionuclides is determined. Since their halflives must be rather long ( $>1 \mathrm{kyr}$ ) in order to cover periods much longer than the 60 years of NM measurements, conventional decay counting technique becomes increasingly inefficient for long half-life radionuclides (Chap. 15 in Beer et al. 2011). Today ${ }^{14} \mathrm{C}$ and ${ }^{10} \mathrm{Be}$ are almost exclusively measured using the accelerator mass spectrometry (AMS) technique. This technique accelerates the atoms of the sample to MeV energies, which leads to the destruction of background molecules and excellent discrimination against isobars $\left({ }^{10} \mathrm{~B}\right.$ in the case of ${ }^{10} \mathrm{Be}$, and ${ }^{14} \mathrm{~N}$ in the case of ${ }^{14} \mathrm{C}$ ), extending the measurable isotopic ratio from typically $\sim 10^{-6}$ for conventional mass-spectrometry, down to $\sim 10^{-14}$ for AMS (Beer 1997).

\section{Temporal Resolution}

The temporal resolution of the paleo-cosmic ray record is determined by the transport processes from the atmosphere into the archives. As noted previously, the ${ }^{10} \mathrm{Be}$ from the troposphere is scavenged within weeks, while contributions from the stratosphere may take up to several years to reach the troposphere. As a consequence the inherent temporal resolution of the ${ }^{10} \mathrm{Be}$ paleo-cosmic ray record is $\sim 1$ to 2 years. Further, the local precipitation rate of snow and rain is crucial in determining the spatial distribution of the nuclides after reaching the troposphere. In summary, the transport and removal of cosmogenic radionuclides from the atmosphere is mainly controlled by the stratosphere-troposphere exchange processes and the spatial distribution of the rain and snow precipitation. Figure 5 shows the ${ }^{10} \mathrm{Be}$ deposition simulated with the ECHAM-HAM global circulation model for the period 1986-1990 (Heikkilä 2007).

Note that the latitude dependence of the deposition flux is completely different from that of the production pattern in Fig. 1. The largest deposition fluxes are observed in the midlatitudes where the majority of the exchange between the stratosphere and the troposphere takes place, and the precipitation rates are large. The model calculations show that as a first approximation, the long-lived cosmogenic radionuclides in the atmosphere can be considered to be well-mixed (Heikkila et al. 2009).

As a general rule it is found that improving the resolution of $\mathrm{a}^{10} \mathrm{Be}$ record to months (by sampling shorter sections of the ice core) leads to a signal which is increasingly dominated by deposition processes. The situation is further complicated by the fact that only a very small fraction of the total amount of produced cosmogenic radionuclides is sampled which, in the case of ${ }^{10} \mathrm{Be}$ and other aerosol borne radionuclides, is then subject to local changes in weather and climate conditions. As will be discussed in Sect. 6, the only way to overcome 


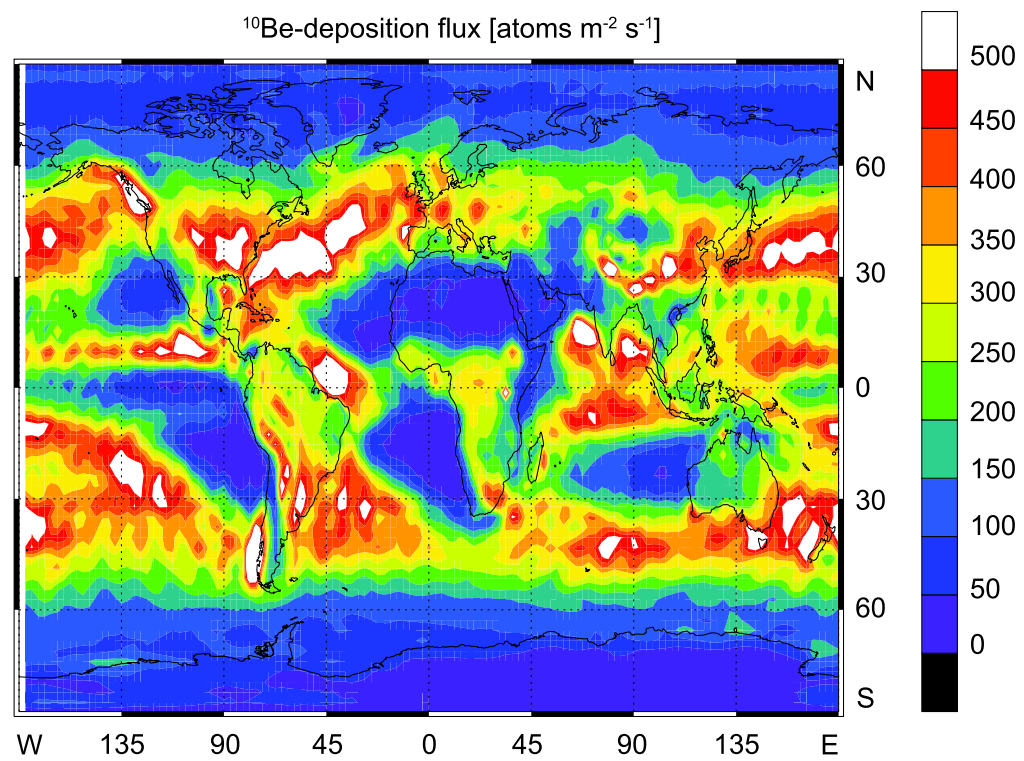

Fig. 5 Simulated mean ${ }^{10} \mathrm{Be}$ deposition flux based on calculated production rates according to (Masarik and Beer 1999) using the ECHAM-HAM general circulation model (Heikkilä 2007)

this difficulty at present is to combine ${ }^{10} \mathrm{Be}$ records from different sites and to extract the common production signal.

For a more detailed discussion of the atmospheric effects we refer to the contribution by Heikkilä et al. (this issue).

Comparing the man-made neutron monitor, and the ${ }^{10} \mathrm{Be}$ paleo-cosmic ray record, we note the following similarities, and differences

1. Both are modulated in a similar manner by heliospheric processes, and the geomagnetic field, with due allowance for the different response functions (Fig. 3).

2. While the neutron monitor measures the integral cosmic ray spectrum above the geomagnetic cut-off rigidity of its location, the ${ }^{10} \mathrm{Be}$ paleo-cosmic ray record responds to the whole of the cosmic ray spectrum above $\sim 100 \mathrm{MeV} /$ nucleon.

3. The inherent temporal resolution of the NM is $<1$ minute, while it is $1-2$ years for the ${ }^{10}$ Be record.

\section{Spatial Dependence}

Instrumental neutron monitors are distributed all over the globe. Each one has its own specific cut-off rigidity and altitude. If a neutron monitor is situated at low geomagnetic latitude the high cut-off rigidity prohibits low rigidity cosmic rays from penetrating into the atmosphere and therefore reduces the amplitude of solar induced modulation. On the other hand, neutron monitors above $70^{\circ}$ geomagnetic latitude are no longer affected by the geomagnetic field and are therefore much more sensitive to solar modulation. This is documented by Fig. 6 which shows the effects of (a) changes of the geomagnetic dipole moment, and (b) changes of the solar modulation potential $\phi$, as a function of the geomagnetic latitude. 
Fig. 6 Relative

latitude-dependence of the ${ }^{10} \mathrm{Be}$ production caused by changes in geomagnetic and solar modulation. A change of the geomagnetic dipole field from 1.5 to 0.5 of the present value causes a production increase of about $55 \%$ for latitudes below $30^{\circ}$. A drop in the solar modulation potential from 900 to $300 \mathrm{MV}$ leads to a similar change at latitudes above $60^{\circ}$.

Atmospheric mixing removes this information almost completely (Chap. 10, Beer et al. 2011)

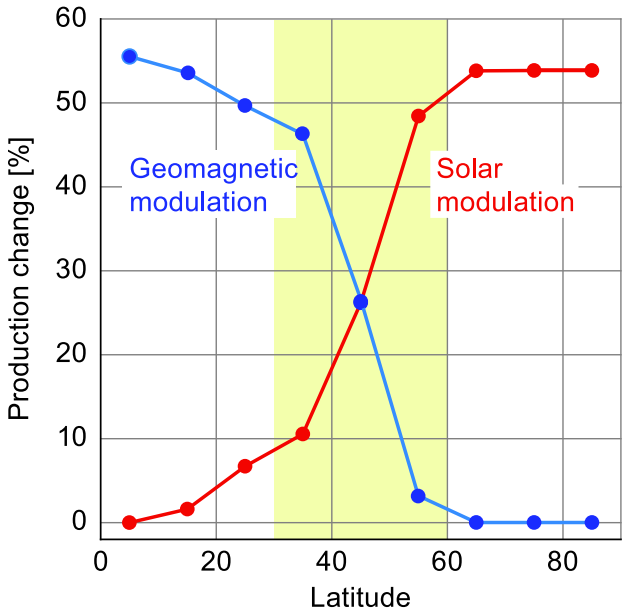

Low latitude neutron monitors are therefore well suited to monitor changes in the geomagnetic field intensity while a high-latitude neutron monitor records solar modulation only. In practice, the geomagnetic dipole field changes very slowly having decreased almost linearly from 11 to $7.8 \times 10^{22} \mathrm{Am}^{2}$ between 1,000 and 2,000 AD.

The time span covered by man-made neutron monitors is therefore much too short to study secular changes in the geomagnetic dipole field. In the case of the natural neutron monitor the situation is completely different. Records of up to 1 million years from ice cores and multi-million years from deep-sea sediments offer a complementary approach to the traditional remanence measurements (Baumgartner et al. 1998; Muscheler et al. 2005; Wagner et al. 2000).

A fundamental difference between man-made and natural neutron monitor is that the latter reflects the global mean production and cannot therefore, a priori, distinguish between heliomagnetic and geomagnetic modulation of cosmic radiation. The main reasons are the complex and relatively slow atmospheric transport and mixing processes discussed in the previous section. It is impossible to trace back a cosmogenic radionuclide stored in an archive to the position in the atmosphere where it has been produced.

\section{Statistical Accuracy of the Paleo-Cosmic Ray Record}

While the modern neutron monitor has a standard deviation of $<0.5 \%$ on hourly data, the

${ }^{10} \mathrm{Be}$ paleo-cosmic ray record has a standard deviation of $\sim 5 \%$ when averaged over 22 years (McCracken et al. 2004). That is, the paleo-cosmic ray record cannot be used for many tasks for which the NM is used- e.g. study of Forbush decreases, the diurnal variation, etc. This has been confirmed recently by the careful comparisons of the $\mathrm{NM}$ and ${ }^{10} \mathrm{Be}$ records made by Webber and Higbie (2010). Both the NM and the paleo-cosmic ray record, however, can be used to study the 11-year Schwabe cycle and long-term variations in the GCR, provided that the striking differences in statistical accuracy and "experimental noise" are recognised. It is stressed that the high accuracy obtained using the NM to study the 11-year cycle will never be attained using the paleo-cosmic ray record; its primary value is to determine the manner in which the 11-year cycles and longer-term variations have varied over the past 10,000 years, and beyond. 
Fig. 7 Comparison between natural and man-made neutron monitors. The solar modulation potential $\phi$ derived from the man-made neutron monitor at Climax is compared to the corresponding $\phi$ values deduced from ${ }^{10} \mathrm{Be}$ concentrations measured in the NGRIP ice core in Greenland (Berggren et al. 2009) after applying a $(1,2,1)$ binomial filter to reduce the transport noise

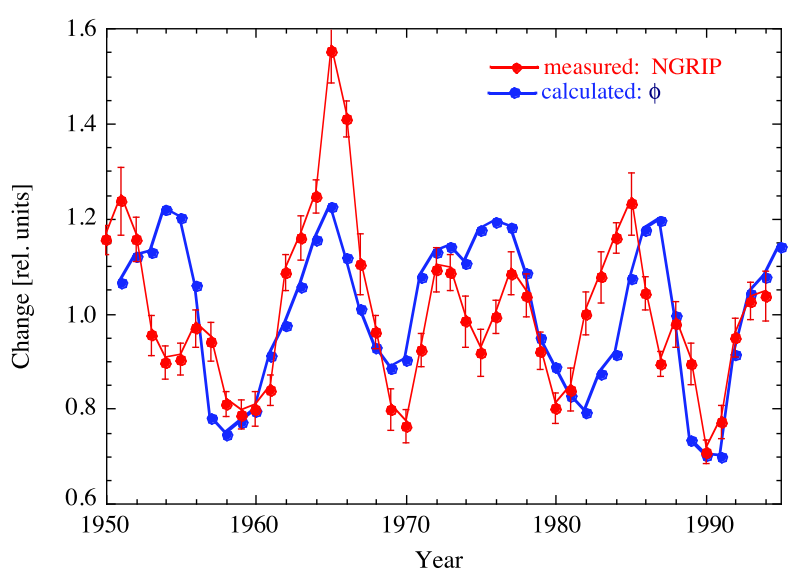

Briefly, the statistical noise in the ${ }^{10} \mathrm{Be}$ record originates as follows. (a) A 5-10\% error on each individual AMS measurement; (b) local variations in the annual snow fall that introduce out of phase variations into the ${ }^{10} \mathrm{Be}$ concentrations; (c) Longer term variations in snowfall, and changes in atmospheric transport effects, resulting in differences in the secular trends in ${ }^{10} \mathrm{Be}$ data from widely separated locations in the north and south polar caps. Using a scatter diagram of $600 \mathrm{yr}$ of 22-year average data from Antarctica and Greenland, McCracken et al. (2004) estimated that the individual 22-year average data sets contained statistical noise with a standard deviation of $\sim 5 \%$. Based on this, we estimate that the standard deviation of annual ${ }^{10} \mathrm{Be}$ data is $\sim 19 \%$, and $\sim 11 \%$ for a $(1,4,6,4,1)$ binomial average of the annual data (a commonly used numerical filter in cosmogenic studies). Bearing in mind that the amplitude of the 11-year cycle in the ${ }^{10} \mathrm{Be}$ paleo-cosmic ray record is $\sim 40 \%$, it is clear that the statistical noise will largely obscure the 11-year cycle in annual data (as shown by Webber and Higbie 2010), while in the case of the $(1,4,6,4,1)$ average, it will distort the 11-year cycle to a considerable degree.

Figure 7 compares the ${ }^{10} \mathrm{Be}$ data from north Greenland, and the data from the Climax neutron monitor. As expected, the $11 \mathrm{yr}$ cycle is evident in the ${ }^{10} \mathrm{Be}$ record, but the phase and amplitude are distorted by the statistical noise.

On the basis that the several sources of statistical and systematic noise outlined above will be uncorrelated between well separated ice cores, we can combine the ${ }^{10} \mathrm{Be}$ records from different sites to extract the common production signal. Principal component analysis (PCA-Jolliffe 2002) is a powerful tool that was first used to this end in 2007 to separate the production signal from system effects (see contribution by Abreu et al. in this issue). An even more satisfactory approach is to combine ${ }^{10} \mathrm{Be}$ ice core records with a ${ }^{14} \mathrm{C}$ tree ring record as in Fig. 8 (Beer et al. 2007). Since both ${ }^{10} \mathrm{Be}$ and ${ }^{14} \mathrm{C}$ are produced in a similar manner, but experience significantly different transport mechanisms from the atmosphere into their respective archives, the common production signal can be extracted using principal component analysis or an equivalent mathematical technique.

\section{The Cosmogenic Record from the Glacial Epoch}

So far, we have discussed the cosmogenic data obtained during the Holocene, the "interglacial" that commenced $\sim 11,000$ y ago. Both the ${ }^{10} \mathrm{Be}$ and ${ }^{14} \mathrm{C}$ records extend back into 
Fig. 8 Comparison of the first component provided by principal component analysis (in green) with the measured relative ${ }^{10} \mathrm{Be}$ (panel a) and ${ }^{14} \mathrm{C}$ (panel b) production changes. The first or production component explains about $90 \%$ of the total variance of the low-pass (1/100 y) filtered data (Beer et al. 2007)

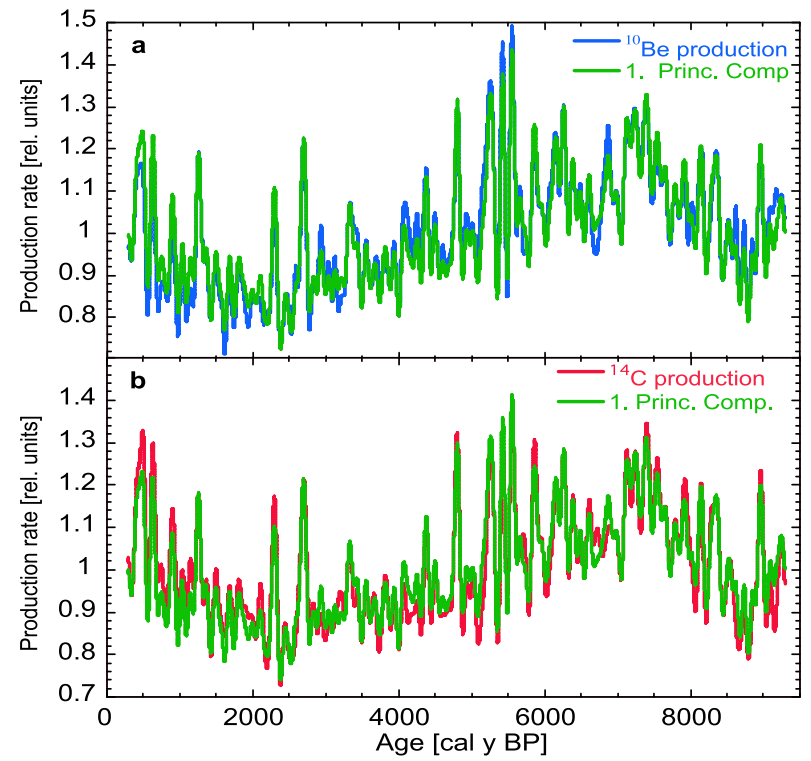

Fig. 9 Comparison between the ${ }^{10} \mathrm{Be}$ concentration and $\delta^{18} \mathrm{O}$ in the GISP 2 ice core (Finkel and Nishiizumi 1997) for the period 3,000-35,000 years BP (BP stands for "Before Present"). Note the jump in ${ }^{10} \mathrm{Be}$ concentration from a relative constant value of $20 \times 10^{3}$ atoms $\mathrm{g}^{-1}$ during the Holocene to values up to $60 \times 10^{3}$ atoms $g^{-1}$ during the last glacial when colder and drier conditions prevailed

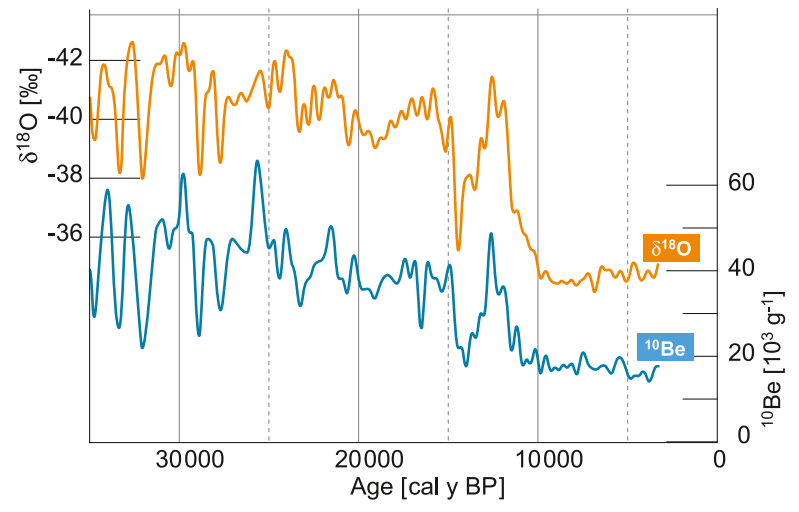

the glacial epoch prior to that date, allowing us to study the heliosphere during that time as well. However there are some complications that we will discuss briefly.

Figure 9 displays the $\delta^{18} \mathrm{O}$ and ${ }^{10} \mathrm{Be}$ concentration in the GISP2 ice core for the past 35,000 years (Finkel and Nishiizumi 1997). $\delta^{18} \mathrm{O}$ (inverse scale) is high for warm temperatures, low for cold. The $\delta^{18} \mathrm{O}$ graph shows that there were persistently low values during the glacial epoch until 15,000 BP. The temperature warmed up for $\sim 1,500$ years, followed by $\sim 1,300$ years (the "Younger Dryas") of temperatures similar to during the glacial epoch, and then about 12,000 BP the temperature started to warm up at the start of the interglacial which continues to this day.

The lower panel of Fig. 9 shows that about $\sim 14,000$ BP the ${ }^{10} \mathrm{Be}$ concentration dropped by a factor of two from a high value $\left(\sim 40 \times 10^{3}\right.$ atoms $\left.\mathrm{g}^{-1}\right)$ to $\sim 20 \times 10^{3}$ atoms g $^{-1}$ (Finkel and Nishiizumi 1997). Figure 9 shows that a short-lived increase to glacial values occurred during the Young Dryas (13,000-11,700 BP). The good correlation between ${ }^{10} \mathrm{Be}$ concentration and $\delta^{18} \mathrm{O}$ clearly points to a causal relationship. Other evidence shows that the water 
cycle was reduced during glacial times, and as a consequence the ice accumulation rate in the polar region was only about $50 \%$ of today's value. That is, the high ${ }^{10} \mathrm{Be}$ concentrations during the last glacial were a direct consequence of the lower precipitation rate at that time. Assuming constant production predicts a decrease in ${ }^{10} \mathrm{Be}$ concentration of a factor of two, as observed between the Younger Dryas and the Holocene.

In practice, analysis of the cosmogenic radionuclides in a sample always commences with a measurement of the concentration; i.e. the number of atoms per gram of the sample material. This is determined by the number of atoms deposited at the sampling site in a year, say, and the amount of precipitation (snow, ice) that these atoms were stored in. Consequently some (but not all) changes in the precipitation rate lead to ${ }^{10} \mathrm{Be}$ concentration changes, which have nothing to do with ${ }^{10} \mathrm{Be}$ production changes. In these cases it may be more appropriate to use the ${ }^{10} \mathrm{Be}$ flux instead of the ${ }^{10} \mathrm{Be}$ concentration. As an example the annual ${ }^{10} \mathrm{Be}$ flux in an ice core is given by

$$
F=\rho a c
$$

with $F$ the ${ }^{10} \mathrm{Be}$ flux in atoms $\mathrm{cm}^{-2} \mathrm{y}^{-1}, \rho$ the density of ice $\left(0.92 \mathrm{~g} \mathrm{~cm}^{-3}\right), a$ the ice accumulation rate in $\mathrm{cm} \mathrm{y}^{-1}$, and $c$ the ${ }^{10} \mathrm{Be}$ concentration in atoms $\mathrm{g}^{-1}$. The ice accumulation rate $\rho$ is measured from other properties of the ice core.

In practice, it is found that neither concentrations nor fluxes are perfect measures of the production rate, and that it depends on the specific conditions whether concentration or flux is preferable. The fact that neither concentration nor flux reflects the production rate correctly is one of the limiting factors of the cosmogenic radionuclide method. In many cases, however, the production changes are large enough (e.g., the Grand Minimum response) that the investigations of interest can be made using either concentration or flux. In addition, during the Holocene the accumulation rate has been rather constant which means that both concentration and flux lead to almost identical production changes.

\section{Conclusions}

Cosmogenic radionuclides provide records of the paleo-cosmic radiation that extend $>10,000$ years into the past. These records were primarily produced by secondary neutrons generated by the galactic cosmic radiation, and can be regarded, in a sense, as providing an extrapolation of the neutron monitor era into the past. While these paleo-cosmic ray data are the only means at our disposal to study the heliosphere in the past, their characteristics are quite different from the man-made neutron monitor in several important respects. Thus (1) they are sensitive to somewhat lower cosmic ray energies; (2) their temporal resolution is $\sim 1$ to 2 years, being determined by the rapidity with which they are sequestered in ice, biological, or other archives; (3) the statistical precision for annual data is poor ( $\sim 19 \%)$; however it is quite adequate ( $\sim 5 \%$ for 22 -year averages) to study the large variations $( \pm 40 \%)$ that have occurred in the paleo-cosmic ray record in the past. The data contains "noise" caused by local meteorological effects, and longer-term climate effects. This noise can be significantly reduced by applying principal component analysis to separate these "system" effects from production effects.

Acknowledgements The research was supported by the Swiss National Science Foundation under grant CRSI122-130642 (FUPSOL). The support of the International Space Science Institute, of Bern, Switzerland is gratefully acknowledged. 


\section{References}

S. Baumgartner, J. Beer, J. Masarik, G. Wagner, L. Meynadier, H.A. Synal, Geomagnetic modulation of the Cl-36 flux in the GRIP ice core, Greenland. Science 279(5355), 1330-1332 (1998)

J. Beer, Accelerator mass spectrometry: a new tool in environmental sciences. Nucl. Phys. News 7(2), 15-22 (1997)

J. Beer, K.G. McCracken, J.A. Abreu, U. Heillila, F. Steinhilber, Long-term changes in cosmic rays derived from cosmogenic radionuclides, in Proc. 30th International Cosmic Ray Conference, Merida, Mexico (2007)

J. Beer, K.G. McCracken, R. von Steiger, Cosmogenic Radionuclides: Theory and Applications in the Terrestrial and Space Environments (Springer, Berlin, 2011)

A.M. Berggren, J. Beer, G. Possnert, A. Aldahan, P. Kubik, M. Christl, S.J. Johnsen, J. Abreu, B.M. Vinther, A 600-year annual Be-10 record from the NGRIP ice core, Greenland. Geophys. Res. Lett. 36 (2009)

R.C. Finkel, K. Nishiizumi, Beryllium-10 concentrations in the Greenland ice sheet project 2 ice core from 3-40 ka. J. Geophys. Res. 102(C12), 26699-26706 (1997)

U. Heikkilä, Modeling of the Atmospheric Transport of the Cosmogenic Radionuclides ${ }^{10} \mathrm{Be}$ and ${ }^{7} \mathrm{Be}$ Using the ECHAM5-HAM General Circulation Model (ETH Zürich, Zürich, 2007)

U. Heikkila, J. Beer, J. Feichter, Meridional transport and deposition of atmospheric Be-10. Atmos. Chem. Phys. 9(2), 515-527 (2009)

J.R. Holton, P.H. Haynes, M.E. McIntyre, A.R. Douglass, R.B. Rood, L. Pfister, Stratosphere-troposphere exchange. Rev. Geophys. 33(4), 403-439 (1995)

I.T. Jolliffe, Principal Component Analysis, 2nd edn. (Springer, New York, 2002)

J. Masarik, J. Beer, Simulation of particle fluxes and cosmogenic nuclide production in the Earth's atmosphere. J. Geophys. Res. 104(D10), 12099-012111 (1999)

J. Masarik, J. Beer, An updated simulation of particle fluxes and cosmogenic nuclide production in the Earth's atmosphere. J. Geophys. Res. Atmos. 114 (2009)

K.G. McCracken, Geomagnetic and atmospheric effects upon the cosmogenic Be-10 observed in polar ice. J. Geophys. Res.-Space Phys. 109(A4) (2004)

K.G. McCracken, F.B. McDonald, J. Beer, G. Raisbeck, F. Yiou, A phenomenological study of the long-term cosmic ray modulation, 850-1958 AD. J. Geophys. Res.-Space Phys. 109(A12) (2004)

R. Muscheler, R. Beer, P.W. Kubik, H.A. Synal, Geomagnetic field intensity during the last 60,000 years based on Be-10 and Cl-36 from the Summit ice cores and C-14. Quat. Sci. Rev. 24(16-17), 1849-1860 (2005)

P. Reimer et al., IntCal09 and Marine09 radiocarbon age calibration curves, 0-50,000 years cal BP. Radiocarbon 51(4), 1111-1150 (2009)

T.G. Shepherd, Transport in the Middle Atmosphere. J. Meteorol. Soc. Jpn. 85B, 165-191 (2007)

U. Siegenthaler, J. Beer, Model Comparison of ${ }^{14} \mathrm{C}$ and ${ }^{10} \mathrm{Be}$ Isotope Records, in Secular Solar and Geomagnetic Variations in the Last 10,000 Years, ed. by F.R. Stephenson, W. Wolfendale (Kluwer Academic, Durham, 1988), pp. 315-328

J.A. Simpson, The cosmic ray nucleonic component: the invention and scientific uses of the neutron monitor (Keynote lecture). Space Sci. Rev., 93(1-2), 11-32 (2000)

G. Wagner, J. Masarik, J. Beer, S. Baumgartner, D. Imboden, P.W. Kubik, H.A. Synal, M. Suter, Reconstruction of the geomagnetic field between 20 and $60 \mathrm{kyr}$ BP from cosmogenic radionuclides in the GRIP ice core. Nucl. Instrum. Methods Phys. Res., Sect. B, Beam Interact. Mater. Atoms, 172, 597-604 (2000)

W.R. Webber, P.R. Higbie, A comparison of new calculations of ${ }^{10}$ Be production in the earth's polar atmosphere by cosmic rays with ${ }^{10} \mathrm{Be}$ concentration measurements in polar ice cores between 1939-2005A troubling lack of concordance, paper 1 (2010). http://arxiv.org/abs/1003.4989; and paper 2 with C.W. Webber, http://arxiv.org/abs/1004.2675 